Check for updates

Cite this: RSC Adv., 2017, 7, 38666

\title{
Stepwise study on Janus-like particles fabricated by polymeric mixtures within soft droplets: a Monte Carlo simulation
}

\author{
Yuping Sheng, (ID)*a Li Xia, ${ }^{a}$ Guanzhou Yang, ${ }^{a}$ Yiqing Xia, (D) ac Yong Huang, ${ }^{a}$ \\ Chuanjiang Pan ${ }^{a}$ and Yutian Zhu (D)*b
}

Using Monte Carlo simulations, a stepwise study (from simple to complex) is followed to investigate Janus particles self-assembled from polymer mixtures within soft droplets. When the polymer mixtures are changed from a homopolymer/homopolymer (A/D) mixture to a diblock copolymer/homopolymer $(A B / D)$ mixture and then to a triblock copolymer/homopolymer (ABC/D) mixture, the self-assembled structures transform from classic Janus particles to hierarchical Janus structures with novel hemidomains. The effects of the incompatibility between two components, polymer-solvent interactions and the ratio of two components of the mixture on the Janus structure are systematically examined. It is found that the Janus structure is highly related to the incompatibility between two components of the mixture, while the sizes of the two hemispheres in a Janus particle can be well tuned by adjusting the ratio of the components in the mixture. Moreover, the corresponding incompatibility between two components of the mixture for the transformation point is increased when the mixture is changed from the $A / D$ to the $A B / D$ and then to the $A B C / D$ mixture. More interestingly, it is found that the variation of the aggregation degree of the changed component with time gradually lags behind that of the homopolymer component (D) during the formation of Janus particles when the component in the mixture changes from $A$ to $A B$ and then to $A B C$. The simulation result could provide theoretical guidance for the preparation of designable Janus particles.

Received 3rd June 2017

Accepted 25th July 2017

DOI: $10.1039 / \mathrm{c} 7 \mathrm{ra06190k}$

rsc.li/rsc-advances of block copolymer mixtures with electrostatic interaction ${ }^{20}$ or hydrogen bonding ${ }^{21,22}$ was also an effective method to fabricate Janus micelles. Basically, the above methods need complex processing procedure or additional interactions between the self-assembly units, which limited the application of Janus materials in various areas.

Recently, it was proved that the confined self-assembly of block copolymers or a polymer mixture within emulsion droplets is a facile and practicable approach to generate asymmetric Janus structures..$^{23-30}$ As the solvent evaporated from the emulsion droplet, micro-phase separation of the block copolymers occurs, resulting in the formation of the asymmetric Janus structures. For example, Deng et al. fabricated Janus nanoparticles with a tunable aspect size ratio from the solvent evaporation induced assembly of poly(4-vinylpyridine) (P4VP)based block copolymers within emulsion droplets. ${ }^{31}$ In addition, confining the polymer mixture inside the emulsion droplet is a useful strategy to create Janus nanoparticles due to the microphase-separation of different polymers or different blocks that occurs within the droplets. Kietzke et al. studied the biphasic morphologies from the miniemulsion with the mixtures of two immiscible polymers. ${ }^{32}$ Janus particles with a tunable volume ratio and well-defined structure were prepared in their study. By means of the phase separation of a binary 
blend in an emulsion droplet, i.e. the diblock copolymer polystyrene- $b$-poly(4-vinylpyridine) (PS- $b$-P4VP) and the homopolymer poly(methyl methacrylate) (PMMA), Deng et al. designed a series of unique Janus colloidal particles with hierarchical structures. ${ }^{33}$ It was found that the internal structures of the Janus particles depended on the copolymer composition, solvent selectivity, particle size, and polymer/aqueous solution interfacial property. Although remarkable performances and progress have been achieved for the fabrication of Janus nanoparticles via the confined self-assembly of block copolymers or polymer mixtures in emulsion droplets, there is still a lack of a clear understanding of this method. For example, some fundamental information, such as the evolution of the chain conformation, control factors and the formation mechanism of the Janus particles, is still not very clear.

On the other hand, it has been proved that computer simulation is a powerful tool to study the self-assembly of block copolymers in solutions. ${ }^{34-41}$ In our previous study, we applied a lattice Monte Carlo method to investigate the coassembly of $\mathrm{AB} / \mathrm{BC}$ diblock copolymer mixture in a selected solvent, which predicted a variety of novel Janus micelles with a controllable micellar shape and Janus architecture. ${ }^{40}$ Recently, a simulated annealing method was developed to mimic the self-assembly of block copolymers in an emulsion droplet. ${ }^{31,37,42} \mathrm{Li}$ and co-workers proposed that the annealing method made the solvent environment gradually change from a good solvent to a poor solvent, resulting in the aggregation of block copolymers, which agrees well with the softconfinement of the block copolymers within the emulation droplets. ${ }^{37}$ Combing with the experiments, they also applied this method to examine the self-assembly of P4VP-based block copolymers within emulsion droplets. ${ }^{31}$ In our previous study, we have successfully applied this annealing simulation method to study the self-assembly of ABA triblock copolymers under soft confinement induced by poor solvent. ${ }^{38}$ Thereafter, we extended this method to mimic the self-assembly of $\mathrm{AB}$ diblock copolymers ${ }^{28}$ and ABC triblock copolymers ${ }^{39}$ under the soft confinement. The simulation results imply that both the shape and internal structure of self-assembled nanoparticles can be tuned by the block copolymer properties, interfacial selectivity, and the confinement size.

In this study, the simulated annealing method is also used to investigate the confined self-assembly of polymer mixtures within soft droplet. The solvent environment for polymer mixture is changed from good to poor gradually to mimic the evaporation of organic solvent in emulsion droplet. Three types of polymer mixtures, i.e. homopolymer (A)/homopolymer (D), diblock copolymer (AB)/homopolymer (D) and linear triblock copolymer (ABC)/homopolymer (D), are used to fabricate Janus particles in sequence. Various control parameters, such as the incompatibility between two components, polymer-solvent interactions and the ratio of the components in the mixture, are examined to reveal their influences on Janus structures. Moreover, the average contact numbers, mean square end-to-end distance and the formation pathways of some representative Janus particles are presented to explicate the formation mechanisms of the Janus structure.

\section{Simulation model and method}

Lattice Monte Carlo (MC) method is used in this simulation. This method has been widely employed to examine the selfassembly of block copolymers. ${ }^{\mathbf{4 2 - 4 7}}$ In this study, the coarse grained chains are placed in a cubic lattice of volume $V=L \times$ $L \times L(L=40)$ with periodic boundary conditions imposed in all three directions. Each monomer occupies one lattice site, and then the remaining vacancy of the lattice represents the solvent. Excluded volume interactions are implemented to guarantee that only one monomer occupies one lattice site. Bond crossing is not allowed. The single-site bond fluctuation model $^{48-50}$ is applied in this simulation, so the bond length of the polymer chain is limited as 1 and $\sqrt{2}$. The initial state is from the randomly dispersed polymer molecules. The selfassembly evolves via the exchange between the monomer and the solvent, i.e. a chain monomer is randomly chosen to exchange with the solvent on its 18 nearest neighbors when this exchange neither violates the bond length nor allows bond crossing. The partial-reptation algorithm ${ }^{51}$ is also used in this simulation: when the exchange induces a single break in the chain, the newly generated break will continue to exchange with the following monomers along the chain until the chain reconnects. Whether the tentative exchange is acceptable or rejective is further determined by the Metropolis rule. ${ }^{52}$ The exchange is accepted when the energy change $\Delta E$ is negative or it is accepted with a probability of $p=\exp$ $\left[-\Delta E / k_{\mathrm{B}} T\right]$, where $\Delta E=\sum_{i j} \Delta N_{i j} \varepsilon_{i j}$ is the energy change aroused by the tentative move; $\Delta N_{i j}$ is the number difference for the nearest neighbor pairs of components $i$ and $j$ induced by the exchange; $i$ and $j$ represent $\mathrm{A}, \mathrm{B}, \mathrm{C}, \mathrm{D}$ and $\mathrm{S}$ (solvent), respectively; $\varepsilon_{i j}$ is the reduced interaction of components $i$ and $j ; k_{\mathrm{B}}$ is the Boltzmann constant and it is fixed as 1 in this simulation; $T$ is the reduced temperature. The annealing method is used in this simulation. This method is used to simulate the evaporation of the organic solvent in an emulsion droplet, which induces the polymer to gradually remain in a poor solvent environment and self-assemble into various structures. The inverse temperature $1 / T$ changes from 0 to 0.07 to achieve the annealing process. The annealing rate is set as 0.0002 per step, and $1 / T$ is fixed as 0.07 after 350 steps and then another 650 steps are taken to ensure the final equilibrium state.

In this simulation, the self-assembly of the mixtures of homopolymer/homopolymer (A/D), diblock copolymer/ homopolymer $(\mathrm{AB} / \mathrm{D})$ and triblock copolymer/homopolymer $(\mathrm{ABC} / \mathrm{D})$ within soft droplets are examined respectively. The concentration of the mixture is fixed as $10 \%$ and all the chain lengths are set as le $=18$. The parameters between the same monomers are set as $\varepsilon_{\mathrm{AA}}=\varepsilon_{\mathrm{BB}}=\varepsilon_{\mathrm{CC}}=\varepsilon_{\mathrm{DD}}=\varepsilon_{\mathrm{SS}}=0$. The interaction parameters, i.e. $\varepsilon_{\mathrm{AS}}, \varepsilon_{\mathrm{BS}}, \varepsilon_{\mathrm{CS}}$ and $\varepsilon_{\mathrm{DS}}$, represent the interactions between different blocks and the solvent. $\varepsilon_{\mathrm{DS}}, \varepsilon_{\mathrm{AS}}, \varepsilon_{\mathrm{BS}}$ and $\varepsilon_{\mathrm{CS}}$ are set as positive values to ensure the solvent is poor for all the blocks. 


\section{Results and discussion}

In this study, we applied Monte Carlo simulation to investigate the formation of Janus particles from the self-assembly of polymer mixtures within soft droplets. The self-assemblies of $\mathrm{A} / \mathrm{D}, \mathrm{AB} / \mathrm{D}$ and $\mathrm{ABC} / \mathrm{D}$ polymer mixtures are examined in sequence. For the three kinds of mixtures, the influences of the incompatibility between two components, the polymer-solvent interaction and the ratio of the components in the mixture on the self-assembly of the polymer mixtures are all systematically examined. Then, the average contact numbers, mean square end-to-end distance and formation pathways of some representative Janus particles are presented to illustrate their chain conformation and formation mechanisms.

\subsection{Janus particles from the mixtures of homopolymer/ homopolymer}

Fig. 1 presents a series of self-assembled morphologies from an $\mathrm{A}_{18} / \mathrm{D}_{18}$ mixture as a function of the incompatibility between the $\mathrm{A}$ and $\mathrm{D}$ homopolymers $\left(\varepsilon_{\mathrm{AD}}\right)$. The polymer-solvent interactions, i.e. $\varepsilon_{\mathrm{AS}}$ and $\varepsilon_{\mathrm{DS}}$, are fixed as 3 , and the ratio of $\mathrm{A}$ and $\mathrm{D}$ is fixed as $1: 1$. When $\varepsilon_{\mathrm{AD}}=0$, i.e. A and D blocks are compatible with each other, a spherical mixed particle is formed with A and D blocks mixing homogeneously. As $\varepsilon_{\mathrm{AD}}$ is increased to 0.15 , microphaseseparation between $\mathrm{A}$ and $\mathrm{D}$ occurs, resulting in the formation of a Janus structure. When $\varepsilon_{\mathrm{AD}}$ is further increased from 1 to 5 , Janus particles with two hemispheres are obtained and the shape of the Janus particle changes from spherical to calabashlike. Clearly, the join of the $\mathrm{A}$ and $\mathrm{D}$ hemispheres becomes increasingly small with the increasing $\varepsilon_{\mathrm{AD}}$. As $\varepsilon_{\mathrm{AD}}$ is increased to 6 , the macro-phase separation between A and D occurs, causing the formation of two distinct A and D spheres. Fig. 1 implies that the incompatibility between $\mathrm{A}$ and $\mathrm{D}$ plays an important role in the formation of the Janus structure.

Beside the incompatibility between $\mathrm{A}$ and $\mathrm{D}$, the interfacial selectivity of the flexible confinement space, i.e. $\varepsilon_{\mathrm{AS}}$ and $\varepsilon_{\mathrm{DS}}$, also greatly affects the resulting structures. In Fig. 2a, various selfassembled morphologies from the $\mathrm{A}_{18} / \mathrm{D}_{18}$ mixtures that changed with $\varepsilon_{\mathrm{AS}}$ are presented. Here, the incompatibility between $\mathrm{A}$ and $\mathrm{D}$ is fixed as $\varepsilon_{\mathrm{AD}}=2$ and the $\mathrm{D}$ homopolymersolvent interaction is set as $\varepsilon_{\mathrm{DS}}=3$. The ratio of $\mathrm{A}$ and $\mathrm{D}$ is fixed as $1: 1$. When the solvent has equal selectivity for $\mathrm{A}$ and $\mathrm{D}$, i.e. $\varepsilon_{\mathrm{AS}}=$ $\varepsilon_{\mathrm{DS}}=3$, a classic Janus particle is obtained. As $\varepsilon_{\mathrm{AS}}$ is increased from 4 to 5.3 , the A domain is gradually covered by the $\mathrm{D}$ domain. Thus, mushroom-like Janus particles are observed when $\varepsilon_{\mathrm{AS}}=5$ and $\varepsilon_{\mathrm{AS}}=5.3$. In experiments, Masayoshi et al. synthesized the mushroom-like Janus particles, which were severed as double stimuli-responsive particulate surfactants. ${ }^{53}$ However, when $\varepsilon_{\mathrm{AS}}$ is

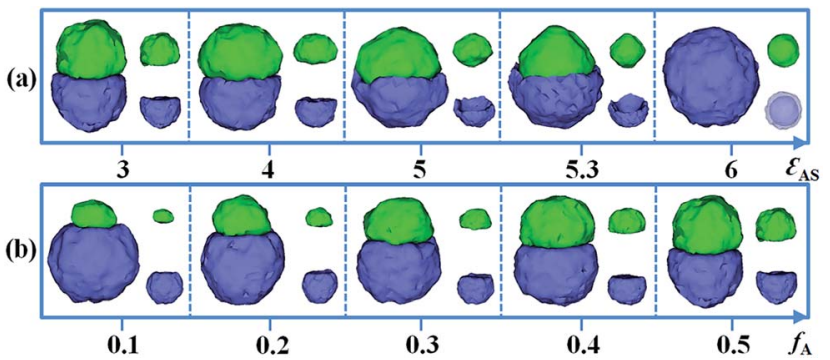

Fig. 2 Self-assembled morphologies of $A_{18} / D_{18}$ mixtures as a function of $\varepsilon_{\mathrm{AS}}(\mathrm{a})$ and $f_{\mathrm{A}}(\mathrm{b})$. The parameter $\varepsilon_{\mathrm{AD}}$ is fixed as 2 . For clarity, the $\mathrm{A}$ and $D$ domains are shown on the right of each corresponding snapshot. The domains of the $D$ blocks are transparent to show the inner structure at $\varepsilon_{\mathrm{AS}}=6$. The color codes of the images are the same as in Fig. 1.

further increased to 6 , an onion-like particle is found instead of the Janus particle. Due to the increased hydrophobicity for $\mathrm{A}_{18}$ homopolymers, the A domain is totally covered by the $\mathrm{D}$ domain to avoid contact with solvent.

The volume fraction of each individual composition also plays an important role on the Janus structure. The influence of $f_{\mathrm{A}}$ (the volume fraction of A homopolymers in the mixture) on the formation of Janus particles is examined. Other parameters are fixed as $\varepsilon_{\mathrm{AS}}=\varepsilon_{\mathrm{DS}}=3$ and $\varepsilon_{\mathrm{AD}}=2$. In Fig. 2b, we show a series of self-assembled morphologies with $f_{\mathrm{A}}$ increased from 0.1 to 0.5 . When $f_{\mathrm{A}}=0.1$, A homopolymers form a small domain on one side, while $\mathrm{D}$ homopolymers aggregate as a big domain on another side, which makes a snowman-shape Janus particle. As $f_{\mathrm{A}}$ is increased from 0.1 to 0.4 , the size of the A hemisphere is gradually increased. When $f_{\mathrm{A}}=0.5$, the $\mathrm{A}$ and $\mathrm{D}$ homopolymers form two equivalent hemispheres, as shown in Fig. 2b. This implies that one can easily tune the size of the two domains of the Janus particles by changing the ratio of the components in the mixtures. In experiments, Deng et $a{ }^{31}{ }^{31}$ successfully tuned Janus structures via adjusting the block ratio of P4VP and PS in the confined self-assembly of PS- $b$-P4VP diblock copolymers within emulsion droplets. Moreover, Kietzke et $a l^{32}$ fabricated the biphasic blend particles with different sizes of polystyrene (PS) and poly(propylene carbonate) (PPC) domains from PS/PPC mixtures by altering the composition ratios. These experimental observations agree well with the morphological transition in our simulations.

\subsection{Janus particles from the mixtures of diblock copolymer/ homopolymer}

In this section, the self-assembly of $\mathrm{AB} / \mathrm{D}$ mixtures is examined to explore more interesting Janus particles. The effect of incompatibility between the diblock copolymer $(\mathrm{AB})$ and the

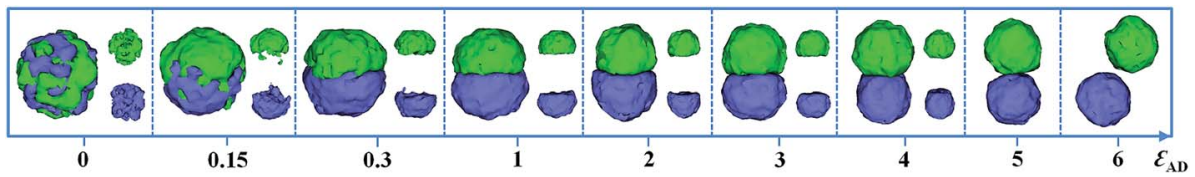

Fig. 1 Self-assembled morphologies of $A_{18} / D_{18}$ mixtures as a function of $\varepsilon_{A D} \cdot \varepsilon_{A S}$ and $\varepsilon_{D S}$ are fixed as 3 . For clarity, the $A$ and $D$ domains are shown on the right of each corresponding snapshot $\left(\varepsilon_{\mathrm{AD}}=0-4\right)$. represents the $\mathrm{A}$ block, while represents the $\mathrm{D}$ block. 


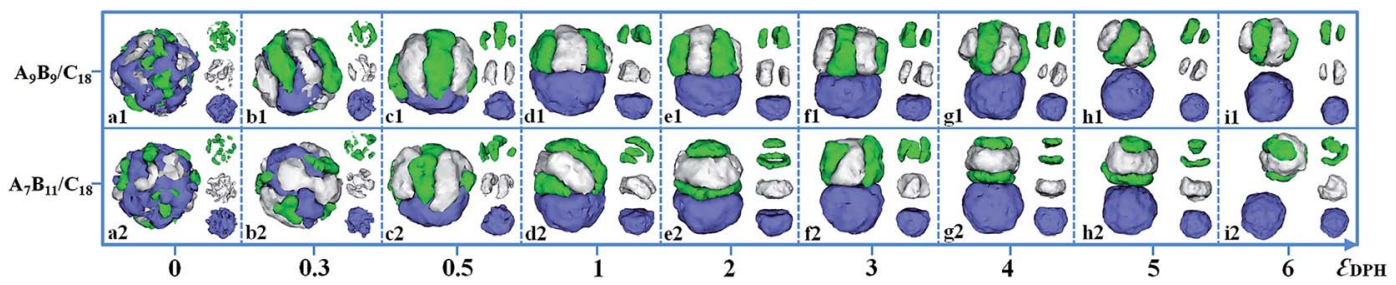

Fig. 3 Self-assembled morphologies of $A_{9} B_{9} / D_{18}$ mixtures and $A_{7} B_{11} / D_{18}$ mixtures as a function of $\varepsilon_{D P H}$. The parameters $\varepsilon_{A S}$, $\varepsilon_{B S}$ and $\varepsilon_{D S}$ are fixed as 3 , and $\varepsilon_{A B}$ is set as 2 . For clarity, the $A, B$ and $D$ domains are shown on the right of each corresponding snapshot. represents the $A$ block, $\square$ represents the $\mathrm{B}$ block and $\mathbf{a}$ represents the $\mathrm{D}$ block.

homopolymer (D) in a mixture (i.e. $\varepsilon_{\mathrm{AD}}$ and $\varepsilon_{\mathrm{BD}}$ ) on the formation of Janus particles is investigated. The incompatibilities between $\mathrm{AB}$ and $\mathrm{D}$ are set as the same value, i.e. $\varepsilon_{\mathrm{AD}}=\varepsilon_{\mathrm{BD}}=\varepsilon_{\mathrm{DPH}}$. Moreover, the incompatibility between the A and B blocks $\left(\varepsilon_{\mathrm{AB}}\right)$ is set as 2 , the parameters of the polymer-solvent interactions, i.e. $\varepsilon_{\mathrm{AS}}, \varepsilon_{\mathrm{BS}}$ and $\varepsilon_{\mathrm{DS}}$, are fixed as 3 , and the ratio of the $\mathrm{AB}$ diblock copolymer and the D homopolymer is fixed as $1: 1$. Fig. 3 shows the selfassembled morphologies of the symmetric diblock copolymer/ homopolymer mixtures $\left(\mathrm{A}_{9} \mathrm{~B}_{9} / \mathrm{D}_{18}\right.$ mixtures) and the asymmetric diblock copolymer/homopolymer mixtures $\left(\mathrm{A}_{7} \mathrm{~B}_{11} / \mathrm{D}_{18}\right)$ as a function of $\varepsilon_{\mathrm{DPH}}$. When the $\mathrm{AB}$ diblock copolymer and the $\mathrm{D}$ homopolymer are compatible with each other, i.e. $\varepsilon_{\mathrm{DPH}}=0$, spherical particles with homogeneously distributed $\mathrm{A}, \mathrm{B}$ and $\mathrm{D}$ domains are formed by the $\mathrm{A}_{9} \mathrm{~B}_{9} / \mathrm{D}_{18}$ mixture (Fig. 3a1). As $\varepsilon_{\mathrm{DPH}}$ is increased to 0.5 , microphase-separation between the $\mathrm{AB}$ diblock copolymers and the $\mathrm{D}$ homopolymers occurs, leading to the $\mathrm{D}$-sphere covered by a segmented AB-shell (Fig. 3b1 and c1). As $\varepsilon_{\mathrm{DPH}}$ is increased from 1 to 3 , further microphase-separation between $A B$ and $D$ occurs, and A and B monomers form segmented hemi-spheres in the Janus particle, as shown in Fig. 3d1-f1. When $\varepsilon_{\mathrm{DPH}}$ is further increased from 4 to 5 , it is observed that the segmented $A B$ hemisphere gradually moves away from the $\mathrm{D}$ hemisphere, as shown in Fig. 3g1-h1. Finally, macroscopic phase separation between $\mathrm{AB}$ diblock copolymers and $\mathrm{D}$ homopolymers is observed when the $\varepsilon_{\mathrm{DPH}}$ is increased to 6 (Fig. 3i1). The morphologies of $\mathrm{A}_{7} \mathrm{~B}_{11} / \mathrm{D}_{18}$ mixtures as a function of the incompatibility between $A B$ and $D$ are also shown in Fig. 3. Similar to $A_{9} B_{9} / D_{18}$ mixtures, a mixed spherical particle is formed by $\mathrm{A}_{7} \mathrm{~B}_{11} / \mathrm{D}_{18}$ mixtures when two components $(\mathrm{AB}$ and $\mathrm{D})$ are compatible with each other
$\left(\varepsilon_{\mathrm{DPH}}=0\right.$, Fig. 3a2). As $\varepsilon_{\mathrm{DPH}}$ is increased from 1 to 5 , various Janus particles with $\mathrm{AB}$ hemispheres and $\mathrm{D}$ hemispheres are observed (Fig. $3 \mathrm{~d} 2-\mathrm{h} 2$ ). When $\varepsilon_{\mathrm{DPH}}$ is further increased to $6, \mathrm{~A}_{7} \mathrm{~B}_{11}$ diblock copolymers and $\mathrm{D}_{18}$ homopolymers formed $\mathrm{AB}$ patchy-like spheres and D spheres, respectively (Fig. 3i2). Fig. 3 indicates that we can create a series of novel Janus particles with $A B$ hemispheres and $\mathrm{D}$ hemispheres via the self-assembly of $\mathrm{AB} / \mathrm{D}$ mixtures within emulsion droplets. Moreover, the internal structures of the $\mathrm{AB}$ hemispheres can be well controlled by the incompatibility between $\mathrm{AB}$ and $\mathrm{D}$.

The effect of polymer-solvent interactions on the formation of Janus particles is also studied in this section. Four types of polymer mixtures, i.e., $\mathrm{A}_{9} \mathrm{~B}_{9} / \mathrm{D}_{18}, \mathrm{~A}_{7} \mathrm{~B}_{11} / \mathrm{D}_{18}, \mathrm{~A}_{6} \mathrm{~B}_{12} / \mathrm{D}_{18}$ and $\mathrm{A}_{5} \mathrm{~B}_{13} /$ $\mathrm{D}_{18}$, are considered in the examination. The incompatibilities between $\mathrm{A}, \mathrm{B}$ and $\mathrm{D}\left(\varepsilon_{\mathrm{AB}}, \varepsilon_{\mathrm{AD}}\right.$ and $\left.\varepsilon_{\mathrm{BD}}\right)$ are set as the same value, i.e. $\varepsilon_{\mathrm{PP}}=2$, and the ratio of the $\mathrm{AB}$ diblock copolymers and the $\mathrm{D}$ homopolymers is fixed as $1: 1$. Fig. 4a shows various Janus particles at different values of $\varepsilon_{\mathrm{PS}}$. The polymer-solvent interactions are set as equal, i.e. $\varepsilon_{\mathrm{AS}}=\varepsilon_{\mathrm{BS}}=\varepsilon_{\mathrm{DS}}=\varepsilon_{\mathrm{PS}}$. For the mixtures of symmetric $\mathrm{A}_{9} \mathrm{~B}_{9}$ diblock copolymers and $\mathrm{D}_{18}$ homopolymers, they self-assemble into Janus particles with segmented $\mathrm{AB}$ hemispheres and D hemispheres when $\varepsilon_{\mathrm{PS}}=3$ or $\varepsilon_{\mathrm{PS}}=4$. As $\varepsilon_{\mathrm{PS}}$ is increased to 5 or 6 , $A$ and $B$ blocks aggregate into the double-helix structure as the $A B$ hemispheres. For the mixture of $A_{7} B_{11} / D_{18}$, a different morphological transition sequence for the $\mathrm{AB}$ hemisphere with an increase of $\varepsilon_{\mathrm{PS}}$ is observed, i.e., the structure transitions from the aggregate of piled disks and rings $\left(\varepsilon_{\mathrm{PS}}=3\right)$ to a twisted-stripe structure $\left(\varepsilon_{\mathrm{PS}}=4\right)$, a double-helix structure $\left(\varepsilon_{\mathrm{PS}}=\right.$ $5)$, and a patchy-like structure $\left(\varepsilon_{\mathrm{PS}}=6\right)$. For the self-assembly of

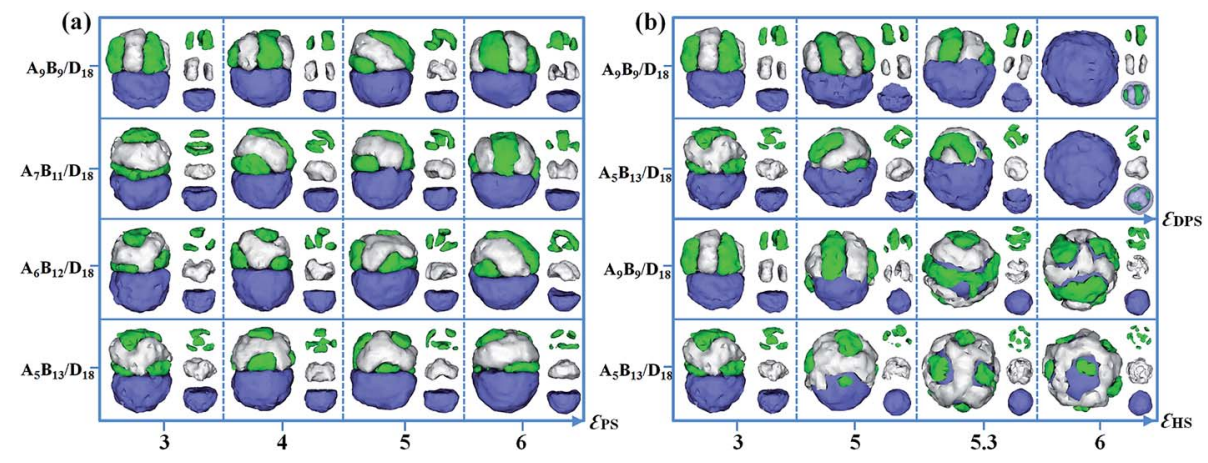

Fig. 4 (a) Self-assembled morphologies of the $A_{9} B_{9} / D_{18}, A_{7} B_{11} / D_{18}, A_{6} B_{12} / D_{18}$ and $A_{5} B_{13} / D_{18}$ mixtures as a function of $\varepsilon_{\text {Ps. }}$ (b) Self-assembled morphologies of $A_{9} B_{9} / D_{18}$ and $A_{5} B_{13} / D_{18}$ mixtures as a function of $\varepsilon_{D P S}$ and $\varepsilon_{H S}$. The parameters $\varepsilon_{P P}$ are fixed as 2 . For clarity, $A, B$ and $D$ domains are shown on the right of each corresponding snapshot. The color codes of the images are the same as in Fig. 3. 


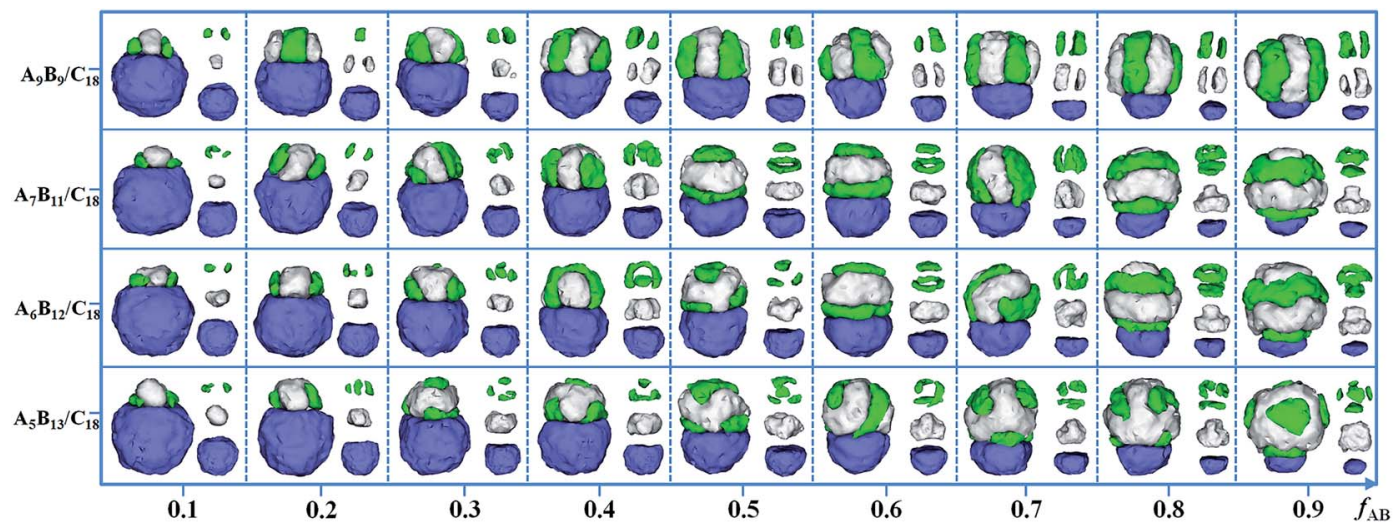

Fig. 5 Self-assembled morphologies of $A_{9} B_{9} / D_{18}, A_{7} B_{11} / D_{18}, A_{6} B_{12} / D_{18}$ and $A_{5} B_{13} / D_{18}$ mixtures as a function of $f_{A B}$. The parameters $\varepsilon_{P S}$ are fixed as 3 , and $\varepsilon_{\mathrm{PP}}$ are fixed as 2 . For clarity, $A, B$ and $D$ domains are shown on the right of each corresponding snapshot. The color codes of the images are the same as in Fig. 3.

$\mathrm{A}_{6} \mathrm{~B}_{12} / \mathrm{D}_{18}$ and $\mathrm{A}_{5} \mathrm{~B}_{13} / \mathrm{D}_{18}$ mixtures, the structures of the $\mathrm{AB}$ hemisphere are independent of $\varepsilon_{\mathrm{PS}}$, and always form the patchylike structures as $\varepsilon_{\mathrm{PS}}$ is increased from 3 to 6 . Furthermore, we also studied the effects of diblock copolymer-solvent interaction $\left(\varepsilon_{\mathrm{DPS}}\right)$ and homopolymer-solvent interaction $\left(\varepsilon_{\mathrm{HS}}\right)$ on the Janus structure from $\mathrm{A}_{9} \mathrm{~B}_{9} / \mathrm{D}_{18}$ and $\mathrm{A}_{5} \mathrm{~B}_{13} / \mathrm{D}_{18}$ mixtures. When the parameter $\varepsilon_{\mathrm{DPS}}$ (or $\varepsilon_{\mathrm{HS}}$ ) is changed from 3 to 6 , the value of $\varepsilon_{\mathrm{HS}}$ (or $\varepsilon_{\mathrm{DPS}}$ ) is fixed as 3. In Fig. $4 \mathrm{~b}$, as $\varepsilon_{\mathrm{DPS}}$ increased from 3 to 5.3 , the $\mathrm{AB}$ domain is gradually covered by the $\mathrm{D}$ domain, inducing the mushroom-like Janus particles. When $\varepsilon_{\mathrm{DPS}}$ is increased to 6 , the $\mathrm{AB}$ domain is totally covered by the $\mathrm{D}$ domain to keep away from solvent. The structure of the $\mathrm{AB}$ domain is kept as a segment aggregate for $\mathrm{A}_{9} \mathrm{~B}_{9} / \mathrm{D}_{18}$ mixtures, while the AB-domain is always a patchy-like structure for $A_{5} B_{13} / D_{18}$ mixtures. Moreover, as $\varepsilon_{H S}$ increases from 3 to 6 , the D domain changes from a hemisphere to a sphere and gradually covers the $\mathrm{AB}$ domain. When $\varepsilon_{\mathrm{DPS}}=5.3$ and 6, the $A_{9} B_{9}$ and $A_{5} B_{13}$ diblock copolymer self-assemble into a stripe-like shell and a patchy-like shell outside the $\mathrm{D}$ sphere.

In order to study the effect of the ratio of two components in a mixture on the formation of Janus particles, the self-assembly of four kinds of mixtures, i.e. $\mathrm{A}_{9} \mathrm{~B}_{9} / \mathrm{D}_{18}, \mathrm{~A}_{7} \mathrm{~B}_{11} / \mathrm{D}_{18}, \mathrm{~A}_{6} \mathrm{~B}_{12} / \mathrm{D}_{18}$ and $A_{5} B_{13} / D_{18}$, are examined in this section. The volume fraction of the $\mathrm{AB}$ diblock copolymers $\left(f_{\mathrm{AB}}\right)$ in the mixture is changed from 0.1 to 0.9 in Fig. 5. Other parameters are fixed, i.e. $\varepsilon_{\mathrm{PS}}=3$ and $\varepsilon_{\mathrm{PP}}$ $=2$. For the mixtures of $A_{9} B_{9} / D_{18}$, the Janus spherical particles form with two different domains on two sides. As $f_{\mathrm{AB}}$ is increased, the morphologies formed by the AB diblock copolymers are always kept as a segmented structure, while the size of the $\mathrm{AB}$ domain and the number of disks gradually increase with $f_{\mathrm{AB}}$. For $\mathrm{A}_{7} \mathrm{~B}_{11} / \mathrm{D}_{18}$ mixtures, the size of the $\mathrm{AB}$ domain in a Janus spherical particle also gradually increases with the increase of $f_{\mathrm{AB}}$, but the morphologies change from segmented structures to aggregates of piled disks and rings. When $f_{\mathrm{AB}}$ is increased in $A_{6} B_{12} / D_{18}$ mixtures, the variation of the $A B$ domain is similar to the morphological change in the mixtures of $A_{7} B_{11} / D_{18}$. The segmented structure, patchy-like structure and aggregate of piled disks and rings are also observed. For $\mathrm{A}_{5} \mathrm{~B}_{13} / \mathrm{D}_{18}$ mixtures, the $\mathrm{AB}$ diblock copolymers form hamburger-like structures at $f_{\mathrm{AB}}=0.1$, and then the A blocks tend to form lumps or strips on the B domain when $f_{\mathrm{AB}}$ is increased from 0.2 to 0.9 . The result illustrates that the structure of the $\mathrm{AB}$ domain in a Janus particle is independent of $f_{\mathrm{AB}}$ for the symmetric copolymer/ homopolymer mixtures, while the AB-structure varied with the increase of $f_{\mathrm{AB}}$ for the asymmetric copolymer/homopolymer mixtures. Moreover, the size of the two domains and the internal structures of the $\mathrm{AB}$ domains can be well tuned by changing the ratio of the two components in the mixture.

\subsection{Janus particles from the mixtures of triblock copolymer/ homopolymer}

In this section, one component in the mixture is changed to a linear triblock copolymer $(\mathrm{ABC})$ while another component is

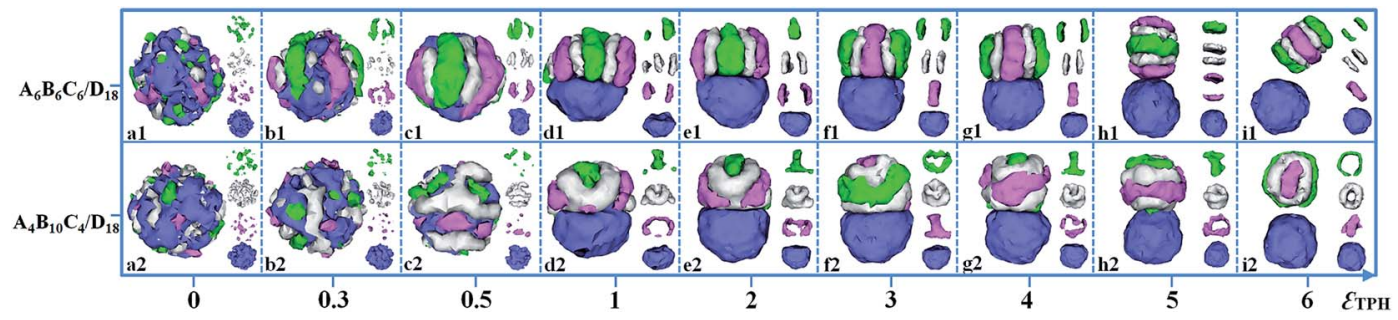

Fig. 6 Self-assembled morphologies of $A_{6} B_{6} C_{6} / D_{18}$ mixtures and $A_{4} B_{10} C_{4} / D_{18}$ mixtures as a function of $\varepsilon_{T P H}$. The parameters $\varepsilon_{A S}, \varepsilon_{B S}, \varepsilon_{C S}$ and $\varepsilon_{D S}$ are fixed as 3 , and $\varepsilon_{\mathrm{AB}}, \varepsilon_{\mathrm{AC}}$ and $\varepsilon_{\mathrm{BC}}$ are set as 2. For clarity, the $\mathrm{A}, \mathrm{B}, \mathrm{C}$ and $\mathrm{D}$ domains are shown on the right of each corresponding snapshot. represents the $\mathrm{A}$ block, $\square$ represents the $\mathrm{B}$ block, $\square$ represents the $\mathrm{C}$ block, and $\square$ represents the $\mathrm{D}$ block. 
fixed as a homopolymer (D). Due to the complex chain architecture, the linear triblock copolymer is expected to exhibit a unique self-assembly behavior in the mixture. In Fig. 6, we examine the effect of incompatibility between the triblock copolymer $(\mathrm{ABC})$ and the homopolymer $(\mathrm{D})$ in a mixture (i.e. $\varepsilon_{\mathrm{AD}}$, $\varepsilon_{\mathrm{BD}}$ and $\left.\varepsilon_{\mathrm{CD}}\right)$ on the formation of Janus particles. The parameter $\varepsilon_{\mathrm{TPH}}$ is used to represent $\varepsilon_{\mathrm{AD}}, \varepsilon_{\mathrm{BD}}$ and $\varepsilon_{\mathrm{CD}}$ because they are set as equal values. Moreover, the parameters $\varepsilon_{\mathrm{AS}}, \varepsilon_{\mathrm{BS}}, \varepsilon_{\mathrm{CS}}$ and $\varepsilon_{\mathrm{DS}}$ are fixed as 3 , and the ratio of the $\mathrm{ABC}$ triblock copolymer and the $\mathrm{D}$ homopolymer is fixed as $1: 1$. The incompatibilities between $\mathrm{A}$, $\mathrm{B}$ and $\mathrm{C}$ blocks, i.e. $\varepsilon_{\mathrm{AB}}, \varepsilon_{\mathrm{AC}}$ and $\varepsilon_{\mathrm{BC}}$, are fixed as 2 . The selfassembled morphologies of $\mathrm{A}_{6} \mathrm{~B}_{6} \mathrm{C}_{6} / \mathrm{D}_{18}$ mixtures (triblock copolymer with equal block length) and $\mathrm{A}_{4} \mathrm{~B}_{10} \mathrm{C}_{4} / \mathrm{D}_{18}$ mixtures (triblock copolymer with relatively long middle block) as a function of $\varepsilon_{\mathrm{TPH}}$ are shown in Fig. 6. When $\mathrm{ABC}$ triblock copolymer is compatible with the D homopolymer, i.e. $\varepsilon_{\mathrm{TPH}}=0$, a spherical particle with homogeneously distributed A, B, C and $D$ domains is formed by the $\mathrm{A}_{6} \mathrm{~B}_{6} \mathrm{C}_{6} / \mathrm{D}_{18}$ blend (Fig. 6a1). As $\varepsilon_{\mathrm{TPH}}$ is increased to 0.5 , microphase-separation between the $\mathrm{ABC}$ triblock copolymers and the D homopolymers occurs, inducing the D sphere to be covered by ABC stripes (Fig. $6 \mathrm{~b} 1$ and $\mathrm{c} 1$ ). As $\varepsilon_{\mathrm{TPH}}$ is increased from 1 to 3 , further microphase-separation between $\mathrm{ABC}$ and $\mathrm{D}$ results in Janus particles with segmented $\mathrm{ABC}$ hemispheres and D hemispheres, as shown in Fig. 6d1-f1. When $\varepsilon_{\mathrm{TPH}}$ is increased from 4 to 5 , the segmented ABC hemisphere gradually parts from the D hemisphere (Fig. 6g1-h1). Then, macroscopic phase separation between the ABC triblock copolymers and the $\mathrm{D}$ homopolymers appears when $\varepsilon_{\mathrm{TPH}}$ is further increased to 6 (Fig. 6i1). The morphologies of the $\mathrm{A}_{4} \mathrm{~B}_{10} \mathrm{C}_{4} / \mathrm{D}_{18}$ mixtures that change with the incompatibility between $\mathrm{ABC}$ and $\mathrm{D}$ are also shown in Fig. 6. Similarly, $\mathrm{A}_{4} \mathrm{~B}_{10} \mathrm{C}_{4} /$ $\mathrm{D}_{18}$ mixtures self-assemble into mixed spherical particles when the two components are compatible with each other $\left(\varepsilon_{\mathrm{TPH}}=0\right.$, Fig. 6a2). As $\varepsilon_{\mathrm{TPH}}$ is increased from 0.3 to 0.5 , microphaseseparation between A, B, C and D occurs, as shown in Fig. 6b2-c2. When $\varepsilon_{\text {TPH }}$ is increased from 1 to 5 , Janus particles with $\mathrm{ABC}$ hemispheres and $\mathrm{D}$ hemispheres are observed, and the $\mathrm{ABC}$ domain gradually moves away from the $\mathrm{D}$ domain, inducing a calabash-like Janus particle (Fig. 6d2-h2). The structure of the $\mathrm{ABC}$ domain is mainly kept as a rod-like structure (A or C) surrounded by a hollow B-lump and strips
(A or C). As $\varepsilon_{\text {TPH }}$ is further increased to 6, a macroscopic phase separation between the ABC triblock copolymers and the D homopolymers appears (Fig. 6i2). Fig. 6 indicates that the Janus structure closely depends on $\varepsilon_{\text {TPH }}$, and different shapes of the Janus particles could be fabricated via adjusting the incompatibilities between $\mathrm{ABC}$ and $\mathrm{D}$.

The effect of polymer-solvent interactions on the formation of Janus particles is examined in this section. Four types of polymer mixtures, i.e., $\mathrm{A}_{6} \mathrm{~B}_{6} \mathrm{C}_{6} / \mathrm{D}_{18}, \mathrm{~A}_{7} \mathrm{~B}_{4} \mathrm{C}_{7} / \mathrm{D}_{18}, \mathrm{~A}_{4} \mathrm{~B}_{10} \mathrm{C}_{4} / \mathrm{D}_{18}$ and $\mathrm{A}_{12} \mathrm{~B}_{3} \mathrm{C}_{3} / \mathrm{D}_{18}$, are considered in the self-assembly. The polymersolvent interactions are set as equal, i.e. $\varepsilon_{\mathrm{AS}}=\varepsilon_{\mathrm{BS}}=\varepsilon_{\mathrm{CS}}=\varepsilon_{\mathrm{DS}}=$ $\varepsilon_{\mathrm{PS}}$, and the ratio of the $\mathrm{ABC}$ diblock copolymers and the $\mathrm{D}$ homopolymers is fixed as $1: 1$. Fig. 7a shows various Janus particles at different values of $\varepsilon_{\mathrm{PS}}$. The mixtures of $\mathrm{A}_{6} \mathrm{~B}_{6} \mathrm{C}_{6}$ triblock copolymers and $\mathrm{D}_{18}$ homopolymers self-assemble into Janus particles with a segmented $\mathrm{ABC}$ hemisphere and a D hemisphere when $\varepsilon_{\mathrm{PS}}$ increases from 3 to 8 . As $\varepsilon_{\mathrm{PS}}$ increases to 10, the $\mathrm{ABC}$ triblock copolymers aggregate into a hemisphere with a patchy-like structure. Similar to the $\mathrm{A}_{6} \mathrm{~B}_{6} \mathrm{C}_{6} / \mathrm{D}_{18}$ mixture, Janus particles with a segmented $\mathrm{ABC}$ hemisphere and a $\mathrm{D}$ hemisphere are also observed from the mixture of $A_{7} B_{4} C_{7} / D_{18}$. But the disks formed by the $\mathrm{B}$ block are relatively thin in the segmented structure due to the short length of the $\mathrm{B}$ block. For the mixture of $\mathrm{A}_{4} \mathrm{~B}_{10} \mathrm{C}_{4} / \mathrm{D}_{18}$, the $\mathrm{A}_{4} \mathrm{~B}_{10} \mathrm{C}_{4}$ triblock copolymers self-assemble into core-shell hemisphere when $\varepsilon_{\mathrm{PS}}=3$. As $\varepsilon_{\mathrm{PS}}$ is increased from 6 to 10 , the hemisphere formed by $\mathrm{A}_{4} \mathrm{~B}_{10} \mathrm{C}_{4}$ is changed into $\mathrm{A}$ and $\mathrm{C}$ particles dispersed on the $\mathrm{B}$ domain. For the self-assembly of $\mathrm{A}_{12} \mathrm{~B}_{3} \mathrm{C}_{3} / \mathrm{D}_{18}$ mixtures, the structures of the $\mathrm{ABC}$ hemisphere are independent of $\varepsilon_{\mathrm{PS}}$, the $\mathrm{B}$ and $\mathrm{C}$ blocks always form Janus lamellae located on the surface of the $A$ domain as $\varepsilon_{\mathrm{PS}}$ is increased from 3 to 10 . Furthermore, the effects of the triblock copolymer-solvent interaction $\left(\varepsilon_{\text {TPS }}\right)$ and homopolymer-solvent interaction $\left(\varepsilon_{\mathrm{HS}}\right)$ on the Janus structures from the $\mathrm{A}_{6} \mathrm{~B}_{6} \mathrm{C}_{6} / \mathrm{D}_{18}$ and $\mathrm{A}_{12} \mathrm{~B}_{3} \mathrm{C}_{3} / \mathrm{D}_{18}$ mixtures are also examined. When the parameter $\varepsilon_{\text {TPS }}$ (or $\varepsilon_{\mathrm{HS}}$ ) is changed from 3 to 6 , the value of $\varepsilon_{\mathrm{HS}}$ (or $\varepsilon_{\mathrm{TPS}}$ ) is fixed as 3 . In Fig. 7b, as $\varepsilon_{\text {TPS }}$ increases from 3 to 5 , the $\mathrm{ABC}$ domain is kept as a segmented structure $\left(\mathrm{A}_{6} \mathrm{~B}_{6} \mathrm{C}_{6}\right)$ or as $\mathrm{BC}$ lumps on an $\mathrm{A}$ aggregate $\left(\mathrm{A}_{12} \mathrm{~B}_{3} \mathrm{C}_{3}\right)$, and gradually covered by the D domain. When $\varepsilon_{\text {TPS }}$ is increased to 6 , the ABC domain is totally covered by the $\mathrm{D}$ domain induced by the strong hydrophobicity of the triblock copolymer. Moreover,

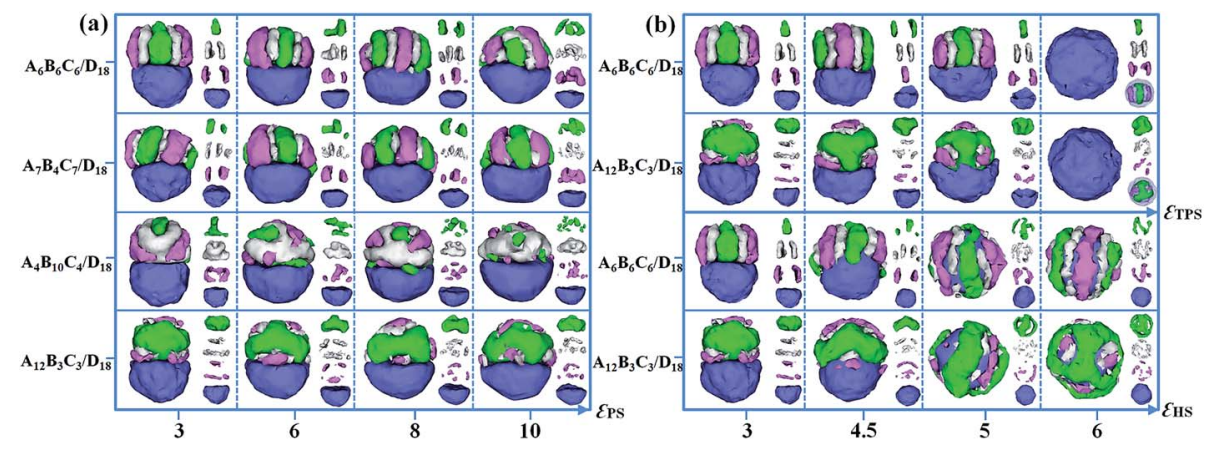

Fig. 7 (a) Self-assembled morphologies of $A_{6} B_{6} C_{6} / D_{18}, A_{7} B_{4} C_{7} / D_{18}, A_{4} B_{10} C_{4} / D_{18}$ and $A_{12} B_{3} C_{3} / D_{18}$ mixtures as a function of $\varepsilon_{P S}$. (b) Selfassembled morphologies of $A_{6} B_{6} C_{6} / D_{18}$ and $A_{12} B_{3} C_{3} / D_{18}$ mixtures as a function of $\varepsilon_{T P S}$ and $\varepsilon_{H S}$. The parameters $\varepsilon_{P P}$ are fixed as 2 . For clarity, the $A, B, C$ and $D$ domains are shown on the right of each corresponding snapshot. The color codes of the images are the same as in Fig. 6 . 
when $\varepsilon_{\mathrm{HS}}$ is increased from 3 to 6 , the D domain transforms from a hemisphere into a sphere and totally covers the $\mathrm{ABC}$ domain after $\varepsilon_{\mathrm{HS}}=5$. The structure of the $\mathrm{A}_{6} \mathrm{~B}_{6} \mathrm{C}_{6}$ domain changes from a segmented structure $\left(\varepsilon_{\mathrm{HS}}=3\right.$ and 4.5$)$ to a stripe-like shell $\left(\varepsilon_{\mathrm{HS}}=5\right)$ and a helix shell $\left(\varepsilon_{\mathrm{HS}}=6\right)$. For the $\mathrm{A}_{12} \mathrm{~B}_{3} \mathrm{C}_{3}$ triblock copolymers, they self-assemble into BC lumps on the A aggregate at $\varepsilon_{\mathrm{HS}}=3$ and $\varepsilon_{\mathrm{HS}}=4.5$, and then aggregate as a patchy-like shell covering the D sphere at $\varepsilon_{\mathrm{HS}}=5$ and $\varepsilon_{\mathrm{HS}}=$ 6.

The influence of $f_{\mathrm{ABC}}$ (the volume fraction of the $\mathrm{ABC}$ triblock copolymers in the mixture) on the formation of the Janus particles is examined through the self-assembly of four kinds of mixtures, i.e. $\mathrm{A}_{6} \mathrm{~B}_{6} \mathrm{C}_{6} / \mathrm{D}_{18}, \mathrm{~A}_{7} \mathrm{~B}_{4} \mathrm{C}_{7} / \mathrm{D}_{18}, \mathrm{~A}_{4} \mathrm{~B}_{10} \mathrm{C}_{4} / \mathrm{D}_{18}$ and $\mathrm{A}_{12} \mathrm{~B}_{3} \mathrm{C}_{3} /$ $\mathrm{D}_{18}$. Other parameters are fixed, i.e. $\varepsilon_{\mathrm{PS}}=3$ and $\varepsilon_{\mathrm{PP}}=2$. The Janus structure changes with $f_{\mathrm{ABC}}$ are shown in Fig. 8. For the mixtures of $\mathrm{A}_{6} \mathrm{~B}_{6} \mathrm{C}_{6} / \mathrm{D}_{18}$, the domains formed by the $\mathrm{ABC}$ triblock copolymers in the Janus spherical particles always remain segmented structures as $f_{\mathrm{ABC}}$ increased from 0.1 to 0.9 . Segmented structures are also observed in $\mathrm{A}_{7} \mathrm{~B}_{4} \mathrm{C}_{7} / \mathrm{D}_{18}$, while the $\mathrm{B}$ disks are thinner than the $\mathrm{A}$ and $\mathrm{C}$ disks. For $\mathrm{A}_{4} \mathrm{~B}_{10} \mathrm{C}_{4} / \mathrm{D}_{18}$ mixtures, the $\mathrm{A}_{4} \mathrm{~B}_{10} \mathrm{C}_{4}$ domain changes from a segmented structure $\left(f_{\mathrm{ABC}}=0.1-0.3\right)$ to an aggregate of rod-like structures (A or C) surrounded by a hollow B-lump and strips (A or C) with the increase of $f_{\mathrm{ABC}}$. When $f_{\mathrm{ABC}}$ is increased in $\mathrm{A}_{12} \mathrm{~B}_{3} \mathrm{C}_{3} / \mathrm{D}_{18}$ mixtures, the structure for $\mathrm{A}_{12} \mathrm{~B}_{3} \mathrm{C}_{3}$ triblock copolymers is kept as $\mathrm{BC}$ lumps on an A aggregate. Fig. 8 shows that the size of the $\mathrm{ABC}$ domain in Janus particles gradually increases with $f_{\mathrm{ABC}}$ for the four kinds of mixtures when $f_{\mathrm{ABC}}$ is increased from 0.1 to 0.9. The result proves that the size of the two domains in Janus particles can be tuned well by changing the volume fraction of compositions in triblock copolymers/homopolymer mixture.

In previous simulations, classic Janus particles, such as Janus-like spheres, cylinders and vesicles, were self-assembled by amphiphile $\mathrm{AB} / \mathrm{BC}$ mixtures in the selected solvent. ${ }^{\mathbf{2 1 , 2 2 , 4 0}}$ These studies proved that the repulsive interaction between the hydrophilic A and $\mathrm{C}$ blocks played a crucial role in the formation of Janus architecture, while the micellar shape was mainly controlled by the solvent quality of the solvophobic blocks. Moreover, Janus particles could be prepared by binary surfactant mixtures absorbed onto the surface of colloid. The combination of elements, colloid size, immiscibility and length of surfactant would determine the formation of various patterns. ${ }^{54-56}$ In addition, the confined self-assembly of a polymer mixture was proved to be a facile method to prepare Janus spheres with two well-defined hemispheres. ${ }^{31,42,57,58}$ The confinement size, the ratio of two components, the volume fraction of blocks and the interactions between different blocks greatly affect the self-assembled morphologies under confinement. In our simulation, it was also indicated that the Janus structure appeared with the appropriate repulsive interaction between two components of the mixture, and too weak or too strong a repulsive interaction induced mixed or separated particles. Through adjusting the solvent quality of one component in the mixture, mushroom-like Janus particles were prepared. The Janus particles with tuned sizes of two domains were achieved by changing the ratio of two components in the mixture. These effects on Janus structures are applicable in the self-assembly of the three kinds of polymer mixtures $(\mathrm{A} / \mathrm{D}, \mathrm{AB} / \mathrm{D}$ and $\mathrm{ABC} / \mathrm{D}$ mixtures).

\subsection{Statistics and comparison}

Clearly, the phase separation between two components dominates the formation of Janus structures, so we calculate the average contact numbers between two components in the mixtures, which can monitor well the microphase-separation degree of the homopolymer (D) and another component (A, $\mathrm{AB}$ or $\mathrm{ABC}$ ). Fig. 9a presents the variation of $N_{\mathrm{AD}}$ (average contact numbers between $\mathrm{A}$ and $\mathrm{D}$ ) of the resulting particles from $\mathrm{A}_{18} / \mathrm{D}_{18}$ mixtures changed with $\varepsilon_{\mathrm{AD}}$. It is observed that $N_{\mathrm{AD}}$ declines rapidly as $\varepsilon_{\mathrm{AD}}$ is increased from 0 to 0.3 , and the morphology changes from a mixed particle to a Janus particle with two hemispheres, as shown in Fig. 1. Thereafter, $N_{\mathrm{AD}}$ decreases slowly when $\varepsilon_{\mathrm{AD}}$ is further increased $\left(0.3<\varepsilon_{\mathrm{AD}}<6\right)$, corresponding to the morphologies transformed from the spherical Janus particle to the calabash-like Janus particle in Fig. 1. It is worth noting that $N_{\mathrm{AD}}$ reaches 0 when $\varepsilon_{\mathrm{AD}}=6$, indicating a macro-phase separation occurs between $\mathrm{A}$ and $\mathrm{D}$. With the application of a linear fit for the data at $\varepsilon_{\mathrm{AD}}<0.3$ and $\varepsilon_{\mathrm{AD}}>0.3$, it is predicted that the transformation point from a mixed particle to a Janus particle is $\varepsilon_{\mathrm{AD}}=0.3$. The variation of $N_{\text {DPH }}$ (average contact numbers between $\mathrm{A}_{9} \mathrm{~B}_{9}$ diblock

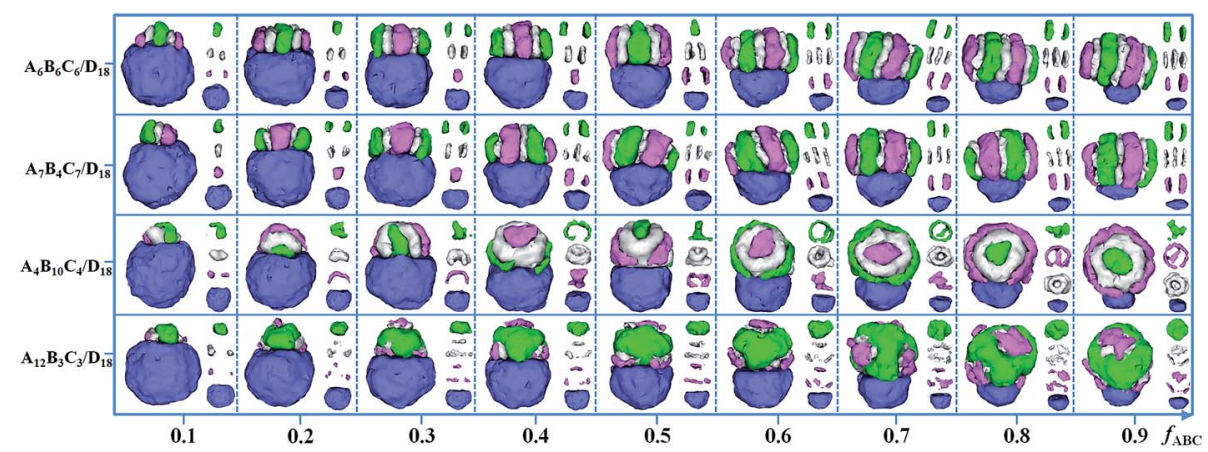

Fig. 8 Self-assembled morphologies of $A_{6} B_{6} C_{6} / D_{18}, A_{7} B_{4} C_{7} / D_{18}, A_{4} B_{10} C_{4} / D_{18}$ and $A_{12} B_{3} C_{3} / D_{18}$ mixtures as a function of $f_{A B C}$. The parameters $\varepsilon_{\mathrm{PS}}$ are fixed as 3 , and $\varepsilon_{\mathrm{PP}}$ are fixed as 2 . For clarity, A, B, C and D domains are shown on the right of each corresponding snapshot. The color codes of the images are the same as in Fig. 6. 

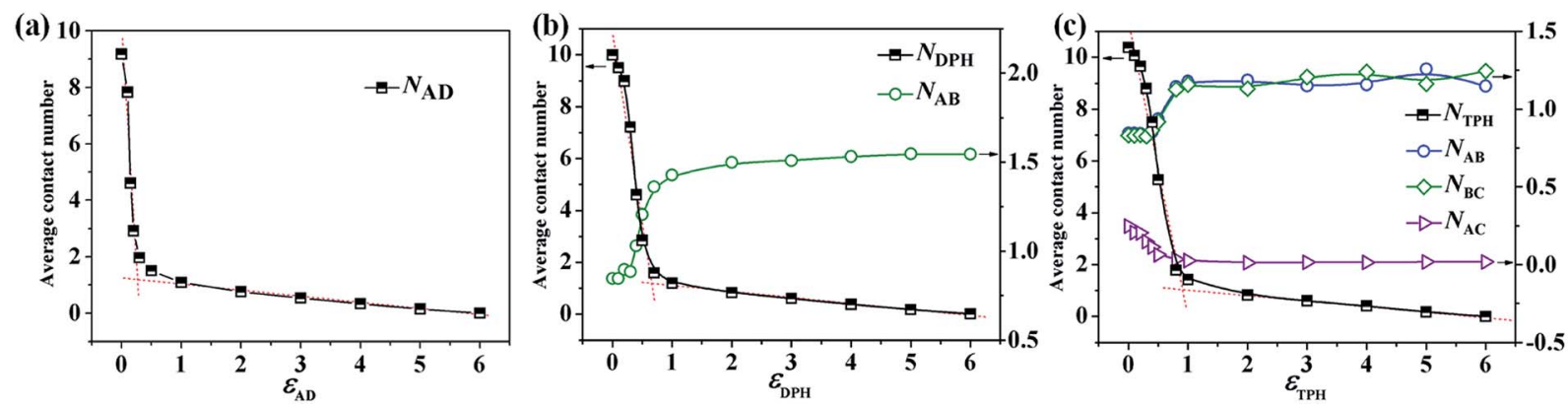

Fig. 9 (a) $N_{A D}$ of the resulting particles of $A_{18} / D_{18}$ mixtures as a function of $\varepsilon_{A D}$; (b) $N_{D P H}$ of the resulting particles of $A_{9} B_{9} / D_{18}$ mixtures as a function of $\varepsilon_{\mathrm{DPH}}, \varepsilon_{\mathrm{AB}}=2$; (c) $N_{\mathrm{TPH}}$ of the resulting particles of $\mathrm{A}_{6} \mathrm{~B}_{6} \mathrm{C}_{6} / \mathrm{D}_{18}$ mixtures as a function of $\varepsilon_{\mathrm{TPH}}, \varepsilon_{\mathrm{AB}}=\varepsilon_{\mathrm{BC}}=\varepsilon_{\mathrm{AC}}=2, \varepsilon_{\mathrm{PS}}=3$.

copolymer and $\mathrm{D}_{18}$ homopolymer) and $N_{\mathrm{AB}}$ (average contact numbers between $\mathrm{A}$ and $\mathrm{B}$ ) of the resulting particles with $\varepsilon_{\mathrm{DPH}}$ are shown in Fig. 9b. The incompatibility between $\mathrm{A}$ and $\mathrm{B}$ is fixed, i.e. $\varepsilon_{\mathrm{AB}}=2$. Similar to $\mathrm{A}_{18} / \mathrm{D}_{18}$ mixtures, $N_{\mathrm{DPH}}$ for $\mathrm{A}_{9} \mathrm{~B}_{9} / \mathrm{D}_{18}$ mixtures changed as $\varepsilon_{\mathrm{DPH}}$ declines rapidly $\left(\varepsilon_{\mathrm{DPH}}<0.7\right)$ and then decreases slowly $\left(\varepsilon_{\mathrm{DPH}}>0.7\right)$, corresponding to the following morphological transition: mixed particles $\rightarrow$ spherical Janus particle $\rightarrow$ calabash-like Janus particle $\rightarrow$ two separated particles (Fig. 3). When we make the linear fit for the curve of $N_{\mathrm{DPH}}$, it is observed that the transformation point from the mixed particle to the Janus particle is $\varepsilon_{\mathrm{DPH}}=0.7$. Moreover, the variation of $N_{\mathrm{AB}}$ with $\varepsilon_{\mathrm{DPH}}$ of the resulting particles selfassembled from the $A_{9} B_{9} / C_{18}$ mixture is also shown in Fig. $9 b$. The curve of $N_{\mathrm{AB}}$ increases rapidly as $\varepsilon_{\mathrm{DPH}}$ is increased from 0 to 0.7. This is attributed to the micro-phase separation between $\mathrm{AB}$ diblock copolymers and $\mathrm{C}$ homopolymers, causing an increase of the contacts between the A and B blocks. And then there is almost no change after the Janus particle forms $\left(\varepsilon_{\mathrm{DPH}}>0.7\right)$, which is induced by the unchanged segmented structure of the $\mathrm{AB}$ domain. In Fig. 9c, the variation of $N_{\mathrm{TPH}}$ (average contact numbers between the $\mathrm{ABC}$ triblock copolymer and the $\mathrm{D}$ homopolymer) changes with $\varepsilon_{\mathrm{TPH}}$ are shown. Similarly, $N_{\mathrm{TPH}}$ declines rapidly at first and then decreases slowly. According to the morphologies shown in Fig. 6a1-i1, the structures changed from mixed particles to Janus particles and then to two separated particles. By means of making a linear fit for the curve of $N_{\text {TPH }}$, the transformation point from a mixed particle to a Janus particle for $\mathrm{A}_{6} \mathrm{~B}_{6} \mathrm{C}_{6} / \mathrm{D}_{18}$ mixtures is $\varepsilon_{\mathrm{TPH}}=1$. Furthermore, the average contact numbers between different polymer monomers for the $\mathrm{A}_{6} \mathrm{~B}_{6} \mathrm{C}_{6}$ triblock copolymers, i.e. $N_{\mathrm{AB}}, N_{\mathrm{BC}}$ and $N_{\mathrm{AC}}$, are also shown in Fig. 9c. It is observed that $N_{\mathrm{AB}}$ and $N_{\mathrm{BC}}$ increase as $\varepsilon_{\mathrm{TPH}}$ is increased from 0 to 1 . This is because the microphaseseparation between $\mathrm{ABC}$ triblock copolymers and $\mathrm{D}$ homopolymers results in an increase of the contacts between the A and $\mathrm{B}$ blocks or between the B and C blocks (Fig. 6a1-d1). The curve for $N_{\mathrm{AC}}$ decreases as $\varepsilon_{\mathrm{TPH}}$ is increased from 0 to 1 , which is induced by the microphase-separation between $\mathrm{A}, \mathrm{B}$ and C. $N_{\mathrm{AC}}$ is decreased to zero because of the formation of an ABCsegmented hemisphere with the $\mathrm{A}$ and $\mathrm{C}$ disks totally divided by the B disk. When $\varepsilon_{\mathrm{TPH}}$ is increased from 1 to $6, N_{\mathrm{AB}}, N_{\mathrm{BC}}$ and $N_{\mathrm{AC}}$ are almost unchanged due to the unchanged segmented structure for the $\mathrm{ABC}$ domain after $\varepsilon_{\mathrm{TPH}}=1$. After comparing the curves of $N_{\mathrm{AD}}, N_{\mathrm{DPH}}$ and $N_{\mathrm{TPH}}$ in Fig. 9, it is found that the transformation point from the mixed particle to the Janus particle exists in all the mixtures, and the corresponding incompatibility between two components $\left(\varepsilon_{\mathrm{AD}}, \varepsilon_{\mathrm{DPH}}\right.$ or $\left.\varepsilon_{\mathrm{TPH}}\right)$ for the transformation point increases from 0.3 to 0.7 and then to 1 when the mixture is changed from $\mathrm{A} / \mathrm{D}$ to $\mathrm{AB} / \mathrm{D}$ and then to $\mathrm{ABC} /$ D mixtures.

Through changing the volume fraction of one component in the mixture, the size of the two domains in self-assembled Janus particles can be tuned well, as shown in Fig. 2 b, 5 and 8 . In this section, the values of the mean square end-to-end distance for the chains of two components in different mixtures $\left(\left\langle R_{\mathrm{X}}{ }^{2}\right\rangle, \mathrm{X}\right.$ represents different polymer chains) are calculated to illustrate the variation of chain conformation. Fig. 10a shows the variation of $\left\langle R_{\mathrm{A}}{ }^{2}\right\rangle$ and $\left\langle R_{\mathrm{D}}{ }^{2}\right\rangle$ of the Janus particles self-assembled by the $\mathrm{A}_{18} / \mathrm{D}_{18}$ mixture changes with $f_{\mathrm{A}}$, corresponding to the morphologies in Fig. 2b. It is observed that $\left\langle R_{\mathrm{A}}{ }^{2}\right\rangle$ is increased with the increase of $f_{\mathrm{A}}$, while $\left\langle{R_{\mathrm{D}}}^{2}\right\rangle$ is decreased with the increase of $f_{\mathrm{A}}$. This indicates that the chains of the A homopolymer stretch with the growing volume fraction in the resulting structure, while the chains of the $\mathrm{D}$ homopolymer become more folded when the size of the $\mathrm{D}$ domain becomes smaller. It is worth noting the curves of $\left\langle R_{\mathrm{A}}{ }^{2}\right\rangle$ and $\left\langle R_{\mathrm{D}}{ }^{2}\right\rangle$ converge at $f_{\mathrm{A}}=0.5$, which illustrates that their stretch degrees are similar when the volume fractions for A and D are the same. Fig. 10b shows the variation of $\left\langle R_{\mathrm{AB}}{ }^{2}\right\rangle$ and $\left\langle{R_{\mathrm{D}}}^{2}\right\rangle$ of the resulting particles self-assembled by $\mathrm{A}_{9} \mathrm{~B}_{9} / \mathrm{D}_{18}$ (Fig. 5) with the increase of $f_{\mathrm{AB}}$. Similar to Fig. 10a, $\left\langle R_{\mathrm{AB}}{ }^{2}\right\rangle$ increases and $\left\langle R_{\mathrm{D}}{ }^{2}\right\rangle$ decreases with the increase of $f_{\mathrm{AB}}$, indicating that $\mathrm{AB}$ diblock copolymers stretch and $\mathrm{D}$ homopolymers fold with growing $f_{\mathrm{AB}}$. Moreover, $\left\langle R_{\mathrm{AB}}{ }^{2}\right\rangle$ is always greater than $\left\langle R_{\mathrm{D}}{ }^{2}\right\rangle$ because the incompatibility between $\mathrm{A}$ and $\mathrm{B}$ makes the chain of the $\mathrm{AB}$ diblock copolymer more extended than the D homopolymer. Fig. 10c shows how the variation of $\left\langle R_{\mathrm{ABC}}{ }^{2}\right\rangle$ and $\left\langle R_{\mathrm{D}}{ }^{2}\right\rangle$ of the Janus particles selfassembled by the $\mathrm{A}_{6} \mathrm{~B}_{6} \mathrm{C}_{6} / \mathrm{D}_{18}$ mixture changed with $f_{\mathrm{ABC}}$ (Fig. 8). $\left\langle R_{\mathrm{ABC}}{ }^{2}\right\rangle$ increases in general with the increase of $f_{\mathrm{AB}}$, indicating that the $\mathrm{ABC}$ triblock copolymer becomes extended as $f_{\mathrm{ABC}}$ increases from 0.1 to $0.9 .\left\langle R_{\mathrm{D}}{ }^{2}\right\rangle$ decreases with the increase of $f_{\mathrm{ABC}}$. Similar to Fig. 10b, the value of $\left\langle R_{\mathrm{ABC}}{ }^{2}\right\rangle$ is always greater than $\left\langle R_{\mathrm{D}}{ }^{2}\right\rangle$ in Fig. 10c because the incompatibility between $\mathrm{A}, \mathrm{B}$ and $\mathrm{C}$ makes the chains more extended. 

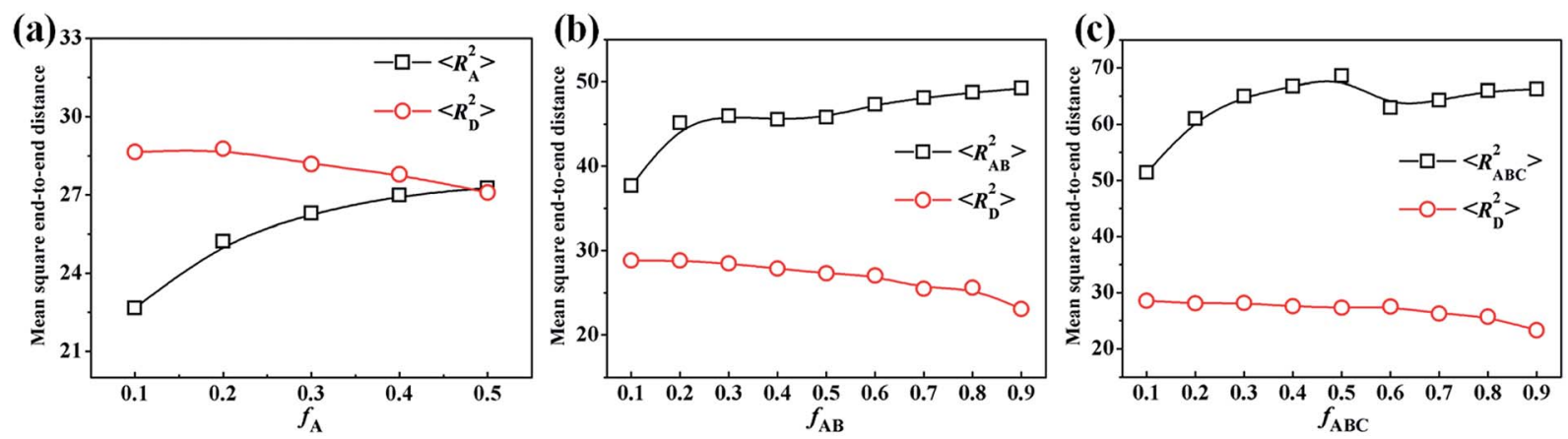

Fig. 10 Mean square end-to-end distance of the chains of the Janus particles self-assembled from an $A_{18} / D_{18}(a), A_{9} B_{9} / D_{18}(b)$ or $A_{6} B_{6} C_{6} / D_{18}(c)$ mixture as a function of $f_{\mathrm{A}}, f_{\mathrm{AB}}$ or $f_{\mathrm{ABC}}$.

The formation pathways of typical Janus particles are investigated to reveal the formation mechanism on the Janus structures. In Fig. 11, $N_{\mathrm{HS}}$ represents the average contact numbers of the homopolymer (D) component with solvent, and $N_{\mathrm{AS}}, N_{\mathrm{DPS}}$ and $N_{\mathrm{TPS}}$ represent another component (A, AB or ABC) with solvent, which can indicate the aggregation degree of the polymer chains. $N_{\mathrm{AH}}, N_{\mathrm{DPH}}$ and $N_{\mathrm{TPH}}$ represent the average contact number between homopolymer (D) and another component (A, $\mathrm{AB}$ and $\mathrm{ABC}$ ) respectively, and $N_{\mathrm{AB}}, N_{\mathrm{AC}}$ and $N_{\mathrm{BC}}$ represent the average contact number between A, B and C-type monomers, indicating the microphase-separation degree between different monomers. For the classic Janus particle with two hemispheres self-assembled by the $\mathrm{A}_{18} / \mathrm{D}_{18}$ mixture (Fig. 1 , $\varepsilon_{\mathrm{AS}}=\varepsilon_{\mathrm{DS}}=3$ and $\left.\varepsilon_{\mathrm{AD}}=2\right)$, the variations of the contact numbers $\left(N_{\mathrm{AS}}, N_{\mathrm{HS}}\right.$ and $\left.N_{\mathrm{AH}}\right)$ with time $(t)$ and the corresponding snapshots at different times are shown in Fig. 11a. The inserted snapshots show that the $\mathrm{A}_{18}$ and $\mathrm{D}_{18}$ homopolymers selfassemble into two loose aggregates separately at first (Fig. 11a2, $t=77$ ), and then these two aggregates keep growing but with two distinct phases (Fig. 11a3, $t=85$ ). As the time is further increased, the aggregates shrink and connect together (Fig. 11a4 and a5, $t=150$ and $t=300$ ), resulting in the formation of the final stable Janus particle (Fig. 11a6).
Moreover, $N_{\mathrm{AS}}$ and $N_{\mathrm{HS}}$ in Fig. 11a show remarkable decreases with time until $t=300$, which implies that homopolymers gradually aggregate into particles. Thereafter, $N_{\mathrm{AS}}$ and $N_{\mathrm{HS}}$ remain almost unchanged with a further increase of time, indicating the aggregation of the $\mathrm{A}$ and $\mathrm{D}$ homopolymers has finished. It is worth noting that the curves of $N_{\mathrm{AS}}-t$ and $N_{\mathrm{HS}^{-}}-t$ completely overlap because of the similar aggregation degree induced by the same polymer-solvent interactions of the A and D homopolymers. The $N_{\mathrm{AH}^{-}}-t$ curve is increased at first due to the rapid aggregation at the beginning (Fig. 11a1, $t=70$ ), and then it starts to decline because of the microphase-separation between A and D (Fig. 11a3, $t=85$ ). Thereafter, $N_{\mathrm{AH}}$ shows a slight increase with time as the $\mathrm{A}$ and $\mathrm{D}$ aggregates shrink gradually (Fig. 11a5, $t=300$ ) and then it keeps almost unchanged as time is further increased.

Fig. 11b shows the formation pathway of Janus particle with a segmented $A B$ hemisphere and $D$ hemisphere from the selfassembly of the $\mathrm{A}_{9} \mathrm{~B}_{9} / \mathrm{D}_{18}$ mixture (Fig. $3, \varepsilon_{\mathrm{DPH}}=2$ ). The variations of the contact numbers, i.e. $N_{\mathrm{DPS}}, N_{\mathrm{HS}}, N_{\mathrm{DPH}}$ and $N_{\mathrm{AB}}$, with $t$ and some typical snapshots at different times are presented to visualize the formation pathways. The inserted snapshots show that $\mathrm{D}_{18}$ homopolymers aggregate into a loose aggregate at first, while $\mathrm{AB}$ diblock copolymers are still uniformly dispersed
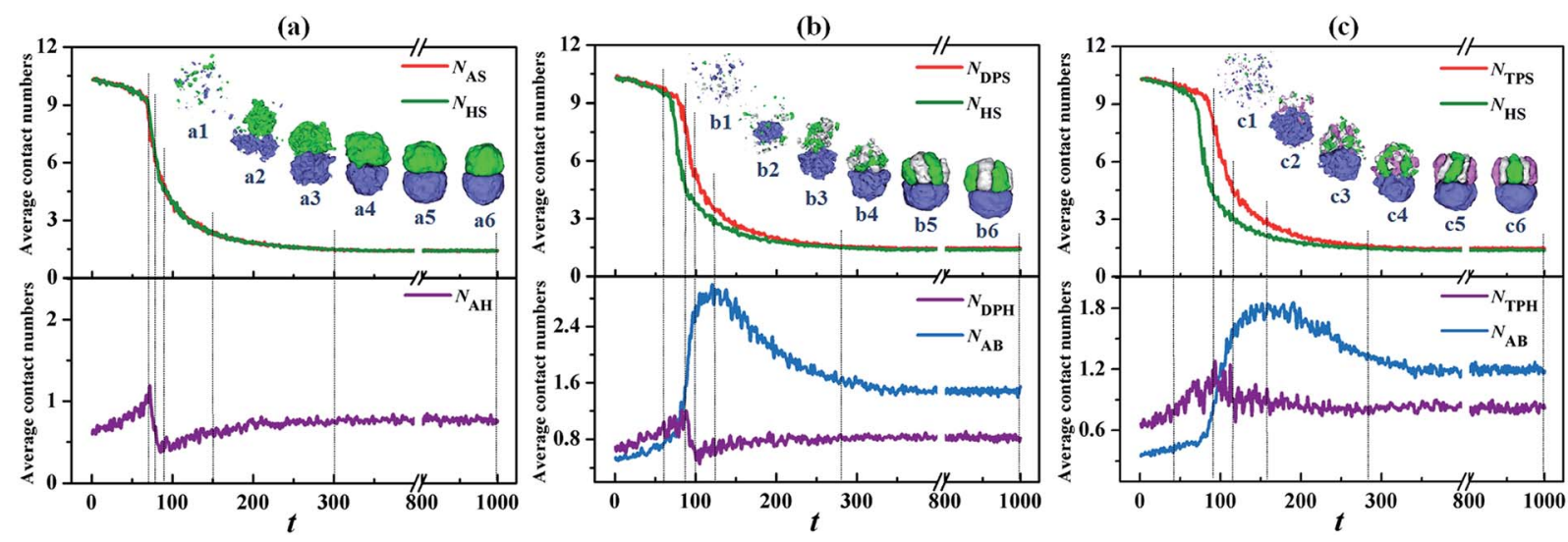

Fig. 11 Variations of the contact numbers with time $(t)$ during the formations of Janus particles self-assembled by the $A_{18} / D_{18}(a), A_{9} B_{9} / D_{18}(b)$, and $A_{6} B_{6} C_{6} / D_{18}$ (c) mixtures. The inserted snapshots show the formation pathway of the Janus particles. The dotted lines correspond to time ( $t$ ) for the inserted snapshots. The color codes of the inserted images are the same as in Fig. 6. 
(Fig. $11 \mathrm{~b} 2, t=85$ ). Maybe this is because the incompatibility between the $\mathrm{A}$ and $\mathrm{B}$ blocks impedes the aggregation of the $\mathrm{AB}$ diblock copolymers. As $t$ increased to 120, the AB diblock copolymers gradually self-assemble into an aggregate, as shown in Fig. $11 \mathrm{~b} 3$ and b4. With a further increase of $t$, microphaseseparation between A and B occurs, resulting in the formation of a segmented structure in the $\mathrm{AB}$ hemisphere (Fig. 11b5, $t=$ 280). Thereafter, the morphology of the Janus particle remains almost unchanged till $t=1000$ (Fig. 11b6). The variations of how the contact numbers changed with $t$ are also shown in Fig. 11b. It is found that the curves of $N_{\mathrm{DPS}}-t$ and $N_{\mathrm{HS}}-t$ obviously decrease until $t=280$ and then remain almost unchanged with an increase in time, which implies that the polymers selfassemble into the aggregate at first and then the aggregate shrinks to form the final particle. However, because of the prior aggregation of the $\mathrm{D}$ homopolymers in the mixture (Fig. 11b2-b4), the decrease of $N_{\mathrm{DPS}}$ lags behind that of $N_{\mathrm{HS}}$ from $t=60$ to $t=280$. The curve of $N_{\mathrm{DPH}^{-}}-t$ increases firstly due to the rapid aggregation at the beginning (Fig. 11b2, $t=85$ ), and then it declines because of the aggregation of the $\mathrm{AB}$ diblock copolymers and the microphase-separation between the $\mathrm{AB}$ and $\mathrm{D}$ domains (Fig. 11b3, $t=100$ ). Then the curve remains almost unchanged with a further increase of $t$. The $N_{\mathrm{AB}}-t$ curve increases remarkably and then declines, corresponding to the aggregation of the mixture (Fig. $11 \mathrm{~b} 4, t=120$ ) and the flowing microphase-separation between A and B (Fig. 11b5, $t=280$ ). Then $N_{\mathrm{AB}}$ remains almost unchanged with the increase of $t$.

Then, the formation pathway of the Janus particle with the segmented $\mathrm{ABC}$ hemisphere and $\mathrm{D}$ hemisphere from the selfassembly of the $\mathrm{A}_{6} \mathrm{~B}_{6} \mathrm{C}_{6} / \mathrm{D}_{18}$ mixture (Fig. $6, \varepsilon_{\text {TPH }}=2$ ) is shown in Fig. 11c. The variations of the contact numbers, $N_{\mathrm{TPS}}, N_{\mathrm{HS}}$, $N_{\mathrm{TPH}}$ and $N_{\mathrm{AB}}$, with $t$ and some typical snapshots at different times are presented. The inserted snapshots show that the $\mathrm{D}_{18}$ homopolymers aggregate into a loose aggregate at first, while for the ABC triblock copolymers some small particles are still dispersed (Fig. 11c2, $t=90$ ). Similar to the aggregate in Fig. 11b2, it is inferred that the incompatibility between the A, B and $\mathrm{C}$ blocks impedes the aggregation of the $\mathrm{ABC}$ triblock copolymers. When $t$ is increased to 120 , the ABC triblock copolymers gradually self-assemble into an aggregate (Fig. 11c3 and c4). As $t$ is further increased, microphase-separation between $\mathrm{A}, \mathrm{B}$ and $\mathrm{C}$ occurs, resulting in the formation of a segmented $\mathrm{ABC}$ hemisphere (Fig. 11c5, $t=280$ ). Then the morphology of the Janus particle remains almost unchanged until $t=1000$ (Fig. 11c6). Fig. 11c also shows the variations of the contact numbers with $t$. It is observed that $N_{\text {TPS }}$ and $N_{\mathrm{HS}}$ show obvious decreases until $t=280$ and then remain almost unchanged with a further increase of $t$. This indicates that the polymer mixture self-assembles into the aggregate and then the aggregate shrinks to form the final Janus particle. However, the prior aggregation of the $\mathrm{D}_{18}$ homopolymers in the mixture (Fig. 11c2-c4) makes the decrease of $N_{\text {TPS }}$ lag behind that of the $N_{\mathrm{HS}}$ from $t=40$ to $t=280$. The curve of $N_{\text {TPH }}$ shows an obvious fluctuation till $t=160$, but it increases firstly and then declines in general. The increase of $N_{\mathrm{TPH}}$ is induced by the rapid aggregation at the beginning (Fig. 11c2, $t=90$ ), and the decrease is because of the aggregation of the $\mathrm{ABC}$ triblock copolymers and the microphase-separation between the $\mathrm{ABC}$ and $\mathrm{D}$ domains (Fig. 11c3, $t=120$ ). Then the curve remains almost unchanged with a further increase of $t$. The variation of $N_{\mathrm{AB}}$ is shown in Fig. 11c; $N_{\mathrm{BC}}$ and $N_{\mathrm{AC}}$ are not shown because the tendencies of their variation are similar to those of $N_{\mathrm{AB}}$. The $N_{\mathrm{AB}}-t$ curve increases obviously and then decreases, corresponding to the aggregation of the polymer mixture (Fig. 11c4, $t=160)$ and the flowing microphase-separation between $\mathrm{A}$ and B (Fig. 11c5, $t=280$ ). Then $N_{\mathrm{AB}}$ remains almost unchanged with an increase of $t$. Through comparing Fig. 11a-c, it is found that all the curves of $N_{\mathrm{HS}}$ show almost the same variation with $t$, while the contact number between another component and the solvent $\left(N_{\mathrm{AS}}, N_{\mathrm{DPS}}\right.$ or $\left.N_{\mathrm{TPS}}\right)$ lags behind $N_{\mathrm{HS}}$ with the growing gap between two curves when the components change from $\mathrm{A}$ to $\mathrm{AB}$ and then to $\mathrm{ABC}$.

\section{Conclusions}

Janus particles self-assembled from different polymer mixtures within soft droplets are investigated using the MC method. The mixtures of homopolymer/homopolymer (A/D) diblock copolymer/homopolymer (AB/D) and triblock copolymer/ homopolymer $(\mathrm{ABC} / \mathrm{D})$ are considered in sequence. The controlled parameters, such as the incompatibility between two components, polymer-solvent interactions and the ratio of the components in the mixture, are systematically examined to illustrate their effects on the formation of Janus particles. The Janus structures are closely related to the incompatibility between two components in the mixture. It is also observed that several mushroom-like Janus particles could be prepared by changing the polymer-solvent interactions, and various Janus particles with tuned sizes of two domains are obtained by adjusting the ratio of two components in a mixture. Both the average contact numbers and mean square end-to-end distance are calculated to explain the chain conformations of the polymers. Furthermore, the formation pathways of some representative Janus particles are shown to illustrate their formation mechanisms. The simulation result is expected to provide theoretical guidance to prepare novel Janus particles.

\section{Conflicts of interest}

There are no conflicts of interest to declare.

\section{Acknowledgements}

This work was financially supported by the National Natural Science Foundation of China (Grants No. 21644013 and 11647029), by the Key Project of Education Department of Sichuan Province (Grant No. 17ZA0283), and by the Scientific Research Project of Sichuan University of Science and Engineering (Grant No. 2015RC32). The simulations were also supported by the High Performance Computing Center, Sichuan University of Science and Engineering. 


\section{References}

1 D. J. Cole-Hamilton, Science, 2010, 327, 41-42.

2 Z. W. Seh, S. Liu, M. Low, S. Y. Zhang, Z. Liu, A. Mlayah and M. Y. Han, Adv. Mater., 2012, 24, 2310-2314.

3 Y. Wang, R. M. Hernandez, D. J. Bartlett, J. M. Bingham, T. R. Kline, A. Sen and T. E. Mallouk, Langmuir, 2006, 22, 10451-10456.

4 T. Nisisako, T. Torii, T. Takahashi and Y. Takizawa, Adv. Mater., 2006, 18, 1152-1156.

5 Z. Yu, C. F. Wang, L. Ling, L. Chen and S. Chen, Angew. Chem., Int. Ed. Engl., 2012, 51, 2375-2378.

6 P.-C. Chen, X. Liu, J. L. Hedrick, Z. Xie, S. Wang, Q.-Y. Lin, M. C. Hersam, V. P. Dravid and C. A. Mirkin, Science, 2016, 352, 1565-1569.

7 N. Glaser, D. J. Adams, A. Böker and G. Krausch, Langmuir, 2006, 22, 5227-5229.

8 J.-W. Kim, D. Lee, H. C. Shum and D. A. Weitz, Adv. Mater., 2008, 20, 3239-3243.

9 A. M. Percebom, J. J. Giner-Casares, N. Claes, S. Bals, W. Loh and L. M. Liz-Marzán, Chem. Commun., 2016, 52, 4278-4281.

10 H. Xing, Z. D. Wang, Z. D. Xu, N. Y. Wong, Y. Xiang, G. L. G. Liu and Y. Lu, ACS Nano, 2012, 6, 802-809.

11 L. Y. Wu, B. M. Ross, S. Hong and L. P. Lee, Small, 2010, 6, 503-507.

12 A. Walther and A. H. E. Müller, Chem. Rev., 2013, 113, 51945261.

13 F. Wurm and A. F. M. Kilbinger, Angew. Chem., Int. Ed., 2009, 48, 8412-8421.

14 A. Walther and A. H. E. Müller, Soft Matter, 2008, 4, 663-668.

15 R. Erhardt, A. Böker, H. Zettl, H. Kaya, W. Pyckhout-Hintzen, G. Krausch, V. Abetz and A. H. E. Müller, Macromolecules, 2001, 34, 1069-1075.

16 A. Walther, X. André, M. Drechsler, V. Abetz and A. H. E. Müller, J. Am. Chem. Soc., 2007, 129, 6187-6198.

17 Y. Liu, V. Abetz and A. H. E. Müller, Macromolecules, 2003, 36, 7894-7898.

18 A. Walther, M. Drechsler, S. Rosenfeldt, L. Harnau, M. Ballauff, V. Abetz and A. H. E. Müller, J. Am. Chem. Soc., 2009, 131, 4720-4728.

19 T. Nisisako, Curr. Opin. Colloid Interface Sci., 2016, 25, 1-12. 20 I. K. Voets, A. de Keizer, P. de Waard, P. M. Frederik, P. H. H. Bomans, H. Schmalz, A. Walther, S. M. King, F. A. M. Leermakers and M. A. Cohen Stuart, Angew. Chem., Int. Ed., 2006, 45, 6673-6676.

21 Y. Han and W. Jiang, J. Phys. Chem. B, 2011, 115, 2167-2172.

22 Y. Han, J. Cui and W. Jiang, J. Phys. Chem. B, 2012, 116, 92089214.

23 R. Deng, F. Liang, J. Zhu and Z. Yang, Mater. Chem. Front., 2017, 1, 431-443.

24 R. Deng, H. Li, F. Liang, J. Zhu, B. Li, X. Xie and Z. Yang, Macromolecules, 2015, 48, 5855-5860.

25 K. H. Ku, Y. Kim, G.-R. Yi, Y. S. Jung and B. J. Kim, ACS Nano, 2015, 9, 11333-11341.

26 J. Xu, Y. Yang, K. Wang, J. Li, H. Zhou, X. Xie and J. Zhu, Langmuir, 2015, 31, 10975-10982.
27 J. Xu, Y. Yang, K. Wang, Y. Wu and J. Zhu, Mater. Chem. Front., 2017, 1, 507-511.

28 N. Yan, Y. Zhu and W. Jiang, J. Phys. Chem. B, 2016, 120, 12023-12029.

29 N. Yan, H. Liu, Y. Zhu, W. Jiang and Z. Dong, Macromolecules, 2015, 48, 5980-5987.

30 R. Deng, F. Liang, X. Qu, Q. Wang, J. Zhu and Z. Yang, Macromolecules, 2015, 48, 750-755.

31 R. Deng, H. Li, J. Zhu, B. Li, F. Liang, F. Jia, X. Qu and Z. Yang, Macromolecules, 2016, 49, 1362-1368.

32 T. Kietzke, D. Neher, M. Kumke, O. Ghazy, U. Ziener and K. Landfester, Small, 2007, 3, 1041-1048.

33 R. H. Deng, S. Q. Liu, F. X. Liang, K. Wang, J. T. Zhu and Z. Z. Yang, Macromolecules, 2014, 47, 3701-3707.

34 J. G. E. M. Fraaije and G. J. A. Sevink, Macromolecules, 2003, 36, 7891-7893.

35 J. Ma, J. Cui, Y. Han, W. Jiang and Y. Sun, $R S C A d v .$, 2015, 5, 86473-86484.

36 J. Fan, J. Cui, Y. Han and W. Jiang, RSC Adv., 2014, 4, 5035150360.

37 P. Chi, Z. Wang, B. Li and A.-C. Shi, Langmuir, 2011, 27, 11683-11689.

38 Y. Sheng, J. An and Y. Zhu, Chem. Phys., 2015, 452, 46-52.

39 N. Yan, Y. Zhu and W. Jiang, Soft Matter, 2016, 12, 965-972.

40 Y. Sheng, X. Yang, N. Yan and Y. Zhu, Soft Matter, 2013, 9, 6254-6262.

41 Y. Han, H. Yu, H. Du and W. Jiang, J. Am. Chem. Soc., 2010, 132, 1144-1150.

42 J. Zhang, W. Kong and H. Duan, Langmuir, 2017, 33, 31233133.

43 Y. Sheng, N. Yan, J. An and Y. Zhu, Chem. Phys., 2014, 441, 47-52.

44 Y. Sheng, Y. Zhu, W. Jiang and Z. Dong, Mater. Chem. Front., 2017, 1, 487-494.

45 Y. Zhu, H. Yu, Y. Wang, J. Cui, W. Kong and W. Jiang, Soft Matter, 2012, 8, 4695-4707.

46 Y. Zhu, X. Yang, W. Kong, Y. Sheng and N. Yan, Soft Matter, 2012, 8, 11156-11162.

47 Y. Zhu and W. Jiang, Macromolecules, 2007, 40, 2872-2881.

48 I. Carmesin and K. Kremer, Macromolecules, 1988, 21, 28192823.

49 R. G. Larson, J. Chem. Phys., 1988, 89, 1642-1650.

50 R. G. Larson, J. Chem. Phys., 1992, 96, 7904-7918.

51 S. Ji and J. Ding, Langmuir, 2005, 22, 553-559.

52 N. Metropolis, A. W. Rosenbluth, M. N. Rosenbluth, A. H. Teller and E. Teller, J. Chem. Phys., 1953, 21, 1087-1092.

53 T. Tanaka, M. Okayama, H. Minami and M. Okubo, Langmuir, 2010, 26, 11732-11736.

54 I. C. Pons-Siepermann and S. C. Glotzer, Soft Matter, 2012, 8, 6226-6231.

55 I. C. Pons-Siepermann and S. C. Glotzer, ACS Nano, 2012, 6, 3919-3924.

56 E. Edlund, O. Lindgren and M. N. Jacobi, Soft Matter, 2014, 10, 2955-2960.

57 R. Yang, B. Li and A.-C. Shi, Langmuir, 2012, 28, 1569-1578.

58 N. Li, A. Z. Panagiotopoulos and A. Nikoubashman, Langmuir, 2017, 33, 6021-6028. 